Article

\title{
Formation and Applications of the Secondary Fiber Bragg Grating ${ }^{\dagger}$
}

\author{
Bai-Ou Guan *, Yang Ran, Fu-Rong Feng and Long Jin \\ Guangdong Provincial Key Laboratory of Optical Fiber Sensing and Communications, Institute of Photonics \\ Technology, Jinan University, Guangzhou 510632, China; rayiori@163.com (Y.R.); ffrhust@126.com (F.-R.F.); \\ iptjinlong@gmail.com (L.J.) \\ * Correspondence: tguanbo@jnu.edu.cn; Tel.: +86-20-8522-0665 \\ † This paper is an invited paper.
}

Academic Editor: Christophe Caucheteur

Received: 15 December 2016; Accepted: 3 February 2017; Published: 18 February 2017

\begin{abstract}
Being one of the most proven fiber optic devices, the fiber Bragg grating has developed continually to extend its applications, particularly in extreme environments. Accompanying the growth of Type-IIa Bragg gratings in some active fibers, a new resonance appears at the shorter wavelength. This new type of grating was named "secondary Bragg grating" (SBG). This paper describes the formation and applications of the SBGs. The formation of the SBG is attributed to the intracore Talbot-type-fringes as a result of multi-order diffractions of the inscribing beams. The SBG presents a variety of interesting characteristics, including dip merge, high-temperature resistance, distinct temperature response, and the strong higher-order harmonic reflection. These features enable its promising applications in fiber lasers and fiber sensing technology.
\end{abstract}

Keywords: fiber Bragg grating; photosensitivity; Talbot effect; distributed Bragg reflector fiber laser; high temperature resistance

\section{Introduction}

Fiber Bragg gratings (FBG) are one of the most well-known optical fiber devices due to their compact size, narrow bandwidth, immunity to electromagnetic interference, and their inherent multiplexing capability [1]. FBGs have been exploited as distributed reflectors in fiber lasers, spectral filters for wavelength-division-multiplexing (WDM) applications, microwave photonic signal processing, and dispersion compensators in optical telecommunications. They are also important photonic sensors, which are capable of measuring temperature [2], strain [3], bending [4], vibration [5], acoustic waves [6], refractive index [7] and, recently, even biomolecules [8]. By assembling and building a wavelength- and/or space-division-multiplexed FBG array, one can monitor the security status of large infrastructures and green energy facilities. The FBG technology has been fast growing in recent years, improving the FBG's performances in tunability, thermal resistance, and lifetime.

A variety of different FBGs have been fabricated with different mechanisms of grating formation $[9,10]$. They have exhibited their own characteristics which enable various applications in different conditions. Type-I FBGs, which are known as common gratings, have positive index modulations and average index changes. The grating formation arises from the changes of the defect polarizability, densification, and stresses. They can stand temperatures below $350{ }^{\circ} \mathrm{C}$.

Type-Ia FBGs are typically formed in hydrogen-loaded fibers. The grating inscription would consume a large amount of cumulative fluence, enabling a greater bulk average index change. A larger red-shift of the Bragg wavelength would occur during inscription. The swelling by hydrogen provides the stress relief through the anisotropic $\mathrm{OH}$ formation. The Type-Ia FBG can withstand the temperature up to $500^{\circ} \mathrm{C}$. 
Regenerated FBG is usually fabricated by post-annealing an over-saturated Type-I FBG written in a heavily hydrogen-loaded fiber. After the full decay of the Type-I seed FBG, a new FBG would regenerate at high temperature, presenting outstanding thermal resistance despite the weak grating strength. The regenerated FBGs have been tested at extremely high temperatures (over $1100{ }^{\circ} \mathrm{C}$ ) with good thermal stability. The grating regeneration is probably attributed to the change of the chemical component of hydroxyl ions at ultra-high temperature.

Type-II FBG is usually fabricated via the ablation of a high-intensity laser beam exceeding the damage threshold of silica. The physical damage-based periodical structure in the silica fiber enhances the thermal stabilization. The Type-II FBG can survive at a temperature of $1000{ }^{\circ} \mathrm{C}$.

The formation of a Type-IIa FBG imparts high demands on the host fiber. The fiber should have a small core diameter, high photosensitivity, and free of hydrogen loading. The Type-IIa FBG would experience a rollover evolution following a saturated Type-I grating. The regrowth resonance presents an obvious blueshift in the reflected wavelength during post-inscription, as a result of the stress relief by the anisotropic relaxation through the dilation of the core-network. Therefore, it is also denoted as "negative-index grating" or "Type-In grating". The Type-IIa FBG can work at temperatures up to $800{ }^{\circ} \mathrm{C}$. It has attracted increasing attention since a new mechanism in photosensitivity needs to be clarified for the negative index changes.

In recent years, Type-IIa FBGs written in different fibers have been reported, including B/Ge (Sn/Ge) co-doped fibers, high Ge-doped fibers, photonic crystal fiber, and microfibers [11-22]. These studies focused on passive fiber-based Type-IIa FBGs. Most recently, we had put forward the study on the Type-IIa FBG directly written in rare-earth doped fibers [23]. The high inscription efficiency endorses the active fiber-based Type-IIa FBG in acting as a strong Bragg reflector, naturally integrated in the laser cavity. More importantly, a new type of grating was discovered, accompanying the formation of the Type-IIa grating. The new type of grating presents a delayed growth and shorter wavelength than the grating which is initially designed. We name this new grating a "secondary Bragg grating" (SBG). In this article, we describe our recent works on SBGs, including the investigation of their formation mechanism, and the applications in high-temperature-resistant fiber grating lasers, thermally-triggered fiber grating lasers, and the higher harmonic grating fiber lasers.

\section{Formation of the SBGs}

The grating inscription setup is shown in Figure 1. A $193 \mathrm{~nm}$ ultraviolet excimer laser (BraggStar Industrial, Coherent. Inc., Santa Clara, CA, USA) is employed for grating inscription. A $120 \mathrm{~mJ} / \mathrm{cm}^{2}$ laser density per pulse is incident upon the fiber after focusing via a cylindrical lens. The repetition rate of the laser is set to $200 \mathrm{~Hz}$. The exposing length is $3 \mathrm{~mm}$, which is determined by the laser beam profile. A standard phase mask with a pitch $\Lambda_{\mathrm{pm}}=1067.17 \mathrm{~nm}$ is adopted. The diffraction efficiencies of the phase mask are $\sim 39 \%$ at first, and $\sim 2 \%$ at second orders, respectively. A broadband light source with a wavelength range of $1500-1600 \mathrm{~nm}$, average power density of $-15 \mathrm{dBm} / \mathrm{nm}$, and an optical spectrum analyzer (OSA) with a resolution of $0.02 \mathrm{~nm}$ (AQ6370C, Yokogawa, Tokyo, Japan) are used to monitor the grating transmission spectrum.

The fiber used here is a commercial photosensitive Er-doped fiber. It has a cladding diameter of $125 \mu \mathrm{m}$ and a core diameter of $3.0 \mu \mathrm{m}$. Due to the heavy germanium dopant in the fiber core, the fiber has a large numerical aperture (N.A.) of 0.24 and a high photosensitivity to UV light. It can meet the inscription requirements of Type-IIa Bragg gratings, considering the small core and high photosensitivity. The cut-off wavelength is $915 \mathrm{~nm}$. The absorption to $980 \mathrm{~nm}$ pump light of the fiber is approximately $5 \mathrm{~dB} / \mathrm{m}$.

The fiber is pre-loaded by a tension of $0.2 \mathrm{~N}$ to accelerate the formation of the Type-IIa grating. Figure 2 shows that the grating spectrum evolves with increasing cumulative UV fluence. It can be seen that the grating grows quickly at first, presenting the evolution of the Type-I grating. Only with $55 \mathrm{~s}$ exposure, the Type-I grating becomes saturated, presenting a reflectivity of $\sim 20 \mathrm{~dB}$. Then the grating starts to decay and eventually achieves a minimal strength of $\sim 4 \mathrm{~dB}$ during another 65 s exposure. 
In the following exposing process, the original primary dip at a longer wavelength regrows with a blue wavelength shift, presenting the Type-IIa grating evolution. Finally, a Type-IIa grating with a reflectivity over $33 \mathrm{~dB}$ is obtained after another six minutes.

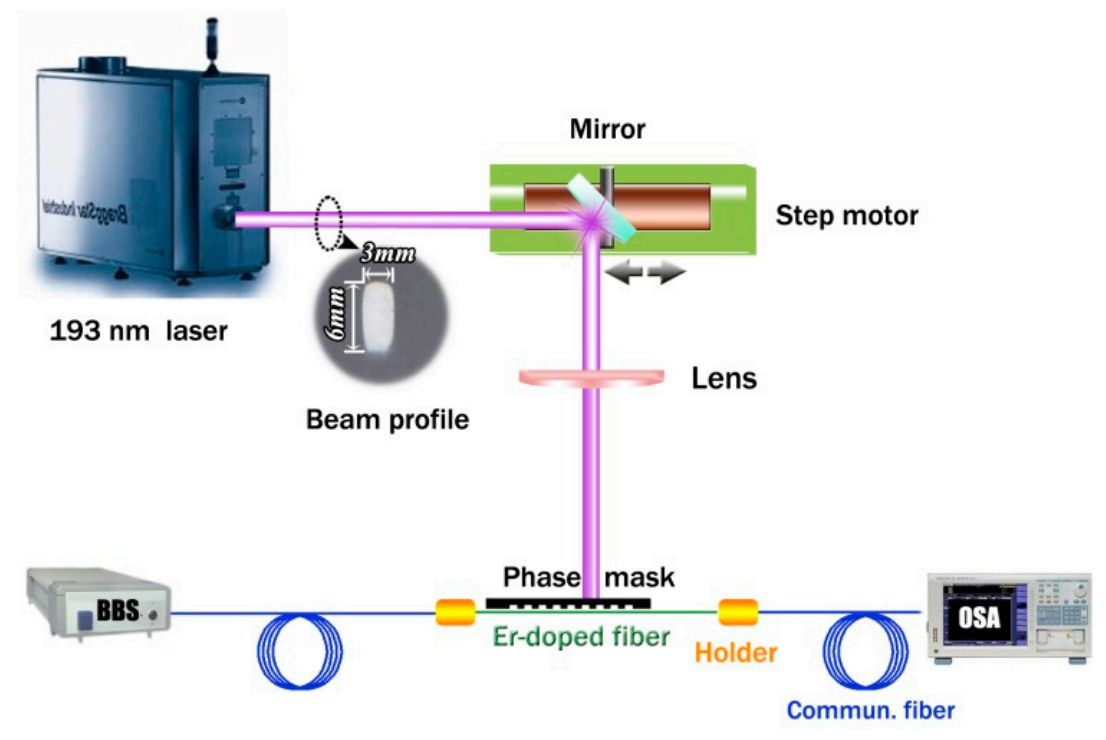

Figure 1. Experimental setup for FBG inscription; BBS: broadband source; OSA: optical spectrum analyzer.

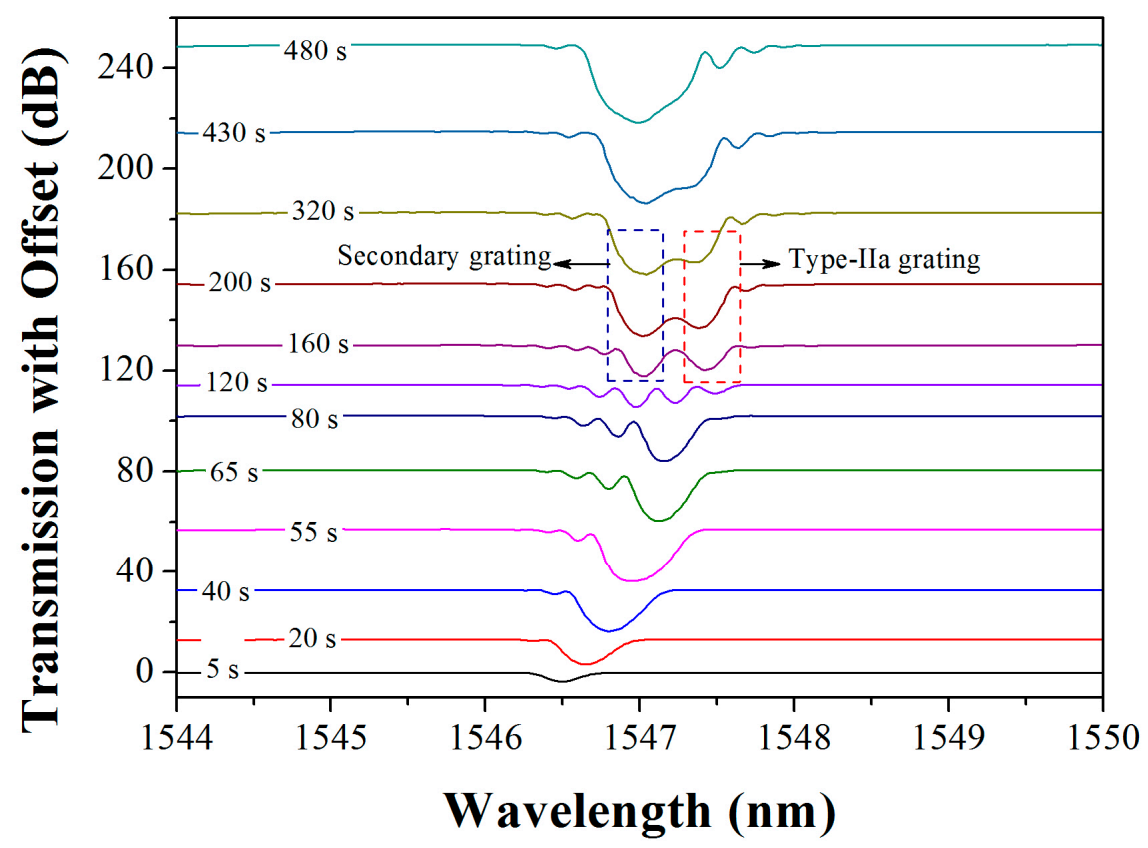

Figure 2. Evolution of the Type-IIa grating and the secondary grating.

Figure 3 illustrates the overall refractive index change of the grating during the inscription. It can be clearly seen that the primary grating experiences a typical "roll-over" evolution process and presents a blue-wavelength-shift at the third stage, in accordance with the formation of the Type-Ila grating.

Here, an abnormal strong dip can be observed at a shorter wavelength with a reflectivity of $10 \mathrm{~dB}$ at the end of the grating decay stage. Moreover, it also grows during the Type-Ila stage and eventually contributes to the strength of the Type-IIa grating. According to the variation of the refractive index 
in Figure 3, the characteristics of this abnormal dip can be revealed. At first, it grows, following the primary dip in Type-I stage, with less index changes both in bulk average and modulation, exhibiting lesser strength at shorter wavelengths. Thus, in order to distinguish it from the primary Bragg grating, we name the dip as the "secondary Bragg grating". Moreover, the secondary dip grows stronger, exceeding the primary dip, which attenuates in the decay stage. In the Type-IIa stage, the secondary dip continues growing, but maintains its wavelength. The Type-Ila dip, which presents a negative index change, approaches the secondary dip gradually. As a sequence, the two dips would integrate through a sufficient cumulative fluence, eventually.

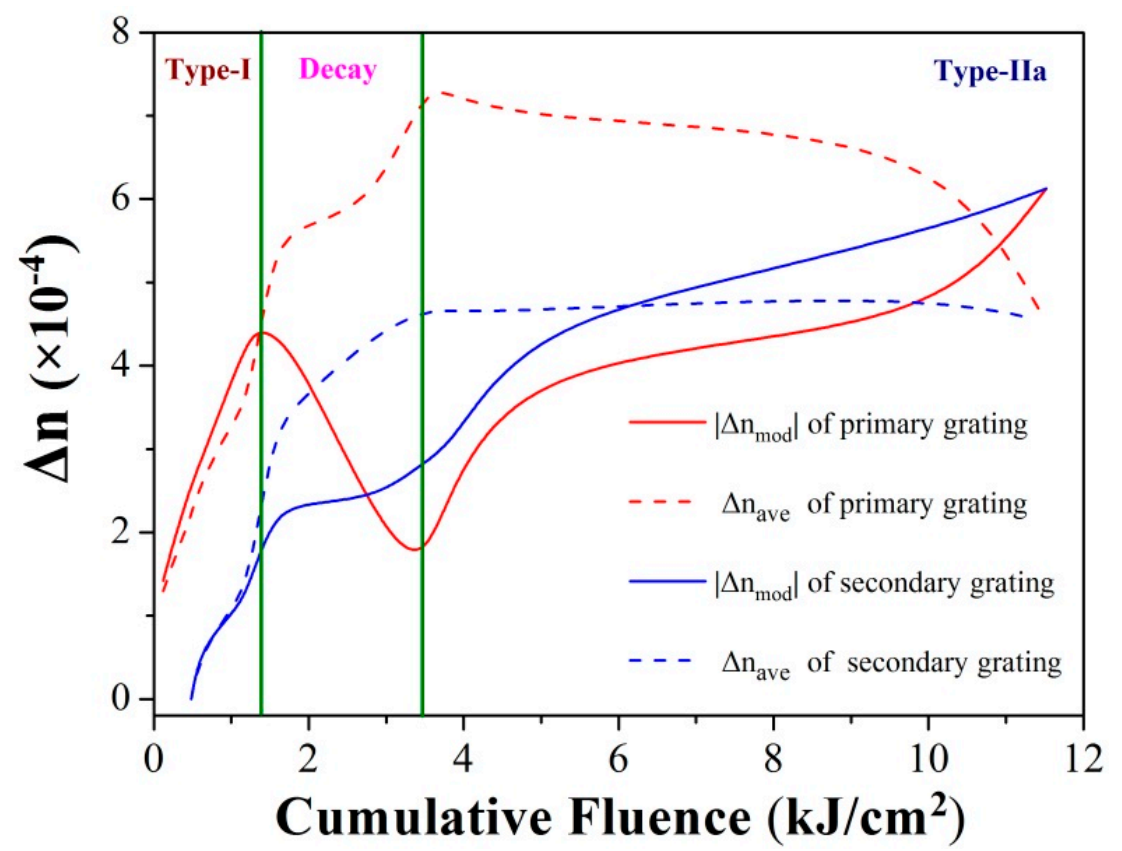

Figure 3. Variation of the indices throughout the grating inscription.

The double-dip phenomenon in the grating is ascribed to the Talbot-type-fringes of the inscription UV laser. For a commercial phase mask, \pm 2 diffraction orders are not fully suppressed $(\sim 2 \%$ remaining in our experiments), affecting the interference fringes formed by the \pm 1 diffraction orders. As a result, Talbot-type fringes are formed in the transversal direction, presenting a sinusoidal variation. The period of the variation is defined as Talbot length. The interleaving transverse index variations with intervals of $\Lambda_{\mathrm{pm}} / 2$ present $\pi$ phase difference [17,24-26].

In our experiment, the Talbot length is estimated to be $6.2 \mu \mathrm{m}$ from the UV laser wavelength and the phase mask period. As the Talbot-type fringes are imprinted in fiber core, the sinusoidal index variation is also formed. Therefore, two second-order-diffraction modulation structures interleaving along the core axis act on the fiber core both with the period of $\Lambda_{\mathrm{pm}}$. By integrating the sinusoidal index variation, the overall effect of the index change (OEIC) can be obtained. Owing to the $\pi$ phase difference of the index variation between those two structures, the OEIC contrast of the two structures is highly dependent to the core diameter. Considering that the diameter of the active fiber here is $3.0 \mu \mathrm{m}$, approximately just the half of the Talbot length, two modulation structures would exhibit significantly OEIC contrast, as shown in Figure 4. The formation of the secondary dip is attributed to the weak influence grating structure by a large amount of exposure of the Type-IIa grating formation, presenting the later growth and shorter wavelength than the primary dip, which is the outcome from the strong-influence grating structure. 


\section{Index modulation}

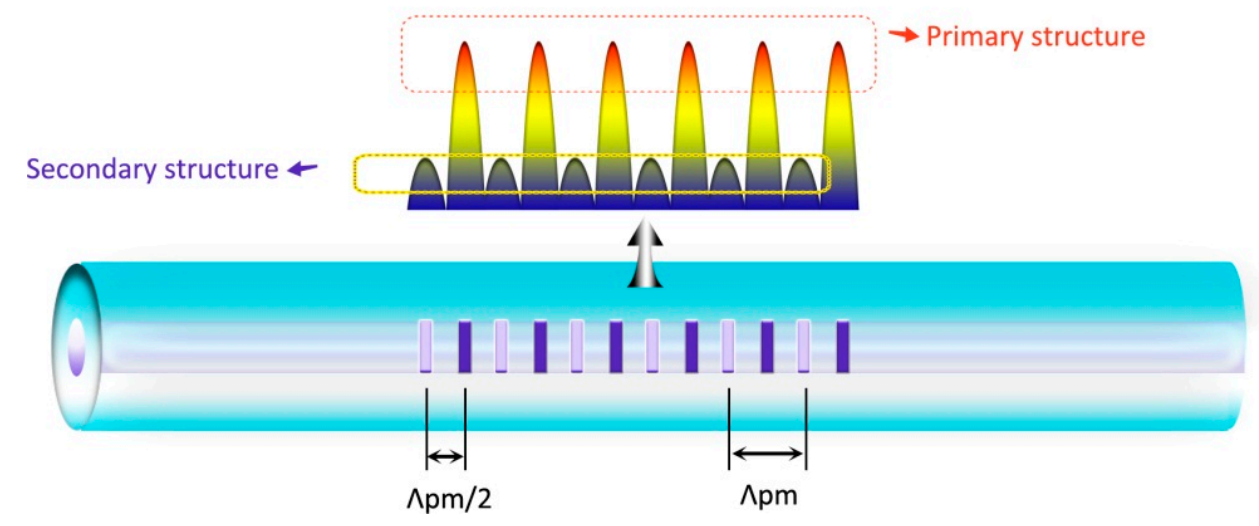

Figure 4. Schematic of the interleaving index-modulation-structures due to the Talbot-type-fringes.

\section{Applications of the SBGs}

The secondary Bragg grating presents a variety of interesting characteristics and shows promising potential in the area of the fiber lasers.

\subsection{High-Temperature-Resistant Short-Cavity DBR Fiber Laser}

It is well known that Type-IIa fiber Bragg gratings exhibit considerable resistance in high-temperature environments without thermal decay. Moreover, the secondary grating would contribute to the whole grating reflectivity as it is integrated with the Type-IIa grating. According to the Figure 2, $33 \mathrm{~dB}$ reflectivity of Type-IIa grating can be obtained by UV exposure within eight minutes. Therefore, we can fabricate a distributed Bragg reflector (DBR) fiber laser through directly inscribing a pair of Typy-IIa gratings into the active fiber as Bragg reflectors. The strong optical feedbacks of the Bragg reflectors can support the laser oscillation within an ultra-short laser cavity.

As shown in Figure 5, we fabricate a Type-IIa grating into the Er-doped fiber with 480 s exposure to produce a stronger reflector. Then the UV beam moves longitudinally by $10 \mathrm{~mm}$. With another $400 \mathrm{~s}$ exposure at the new position, a weaker Type-Ila reflector is fabricated. The entire laser length is $13 \mathrm{~mm}$, including the two Type-IIa gratings. The inset of Figure 5 shows the photograph of Type-IIa grating based DBR fiber laser. The Er-doped fiber emits green fluorescence when excited at $980 \mathrm{~nm}$. The green fluorescence in the grating regions is much weaker than that in the intracavity region. It seems that the formation of Type-IIa grating inhibits the energy transfer upconversion in Er-doped fiber.

Figure 6 shows the transmission spectrum of the Fabry-Perot cavity and the laser output spectrum recorded by an OSA with resolution set to $0.02 \mathrm{~nm}$. Owing to the short cavity length, the longitudinal mode space is $0.1 \mathrm{~nm}$. The laser oscillates at $1546.8 \mathrm{~nm}$ at room temperature. In the experiments, the laser performs a stable single-longitudinal-mode operation, which is a very important quality for applications in sensing and communication systems.

The performance of the DBR laser at high temperature is tested. The laser cavity is placed in a tube oven which can be operated from room temperature to $1200^{\circ} \mathrm{C}$. We recorded the laser output spectrum with one hundred centigrade steps increasing from $200{ }^{\circ} \mathrm{C}$ to $600{ }^{\circ} \mathrm{C}$. The duration under the temperatures below $600{ }^{\circ} \mathrm{C}$ are set to one hour after the stabilization. At $600{ }^{\circ} \mathrm{C}$, we provide a longer time of $2 \mathrm{~h}$. As is shown in Figure 7, the lasing wavelength redshifts along with the temperature enhancement, presenting a highly linear response. The sensitivity of the laser can be calculated as $\sim 12 \mathrm{pm} /{ }^{\circ} \mathrm{C}$. 


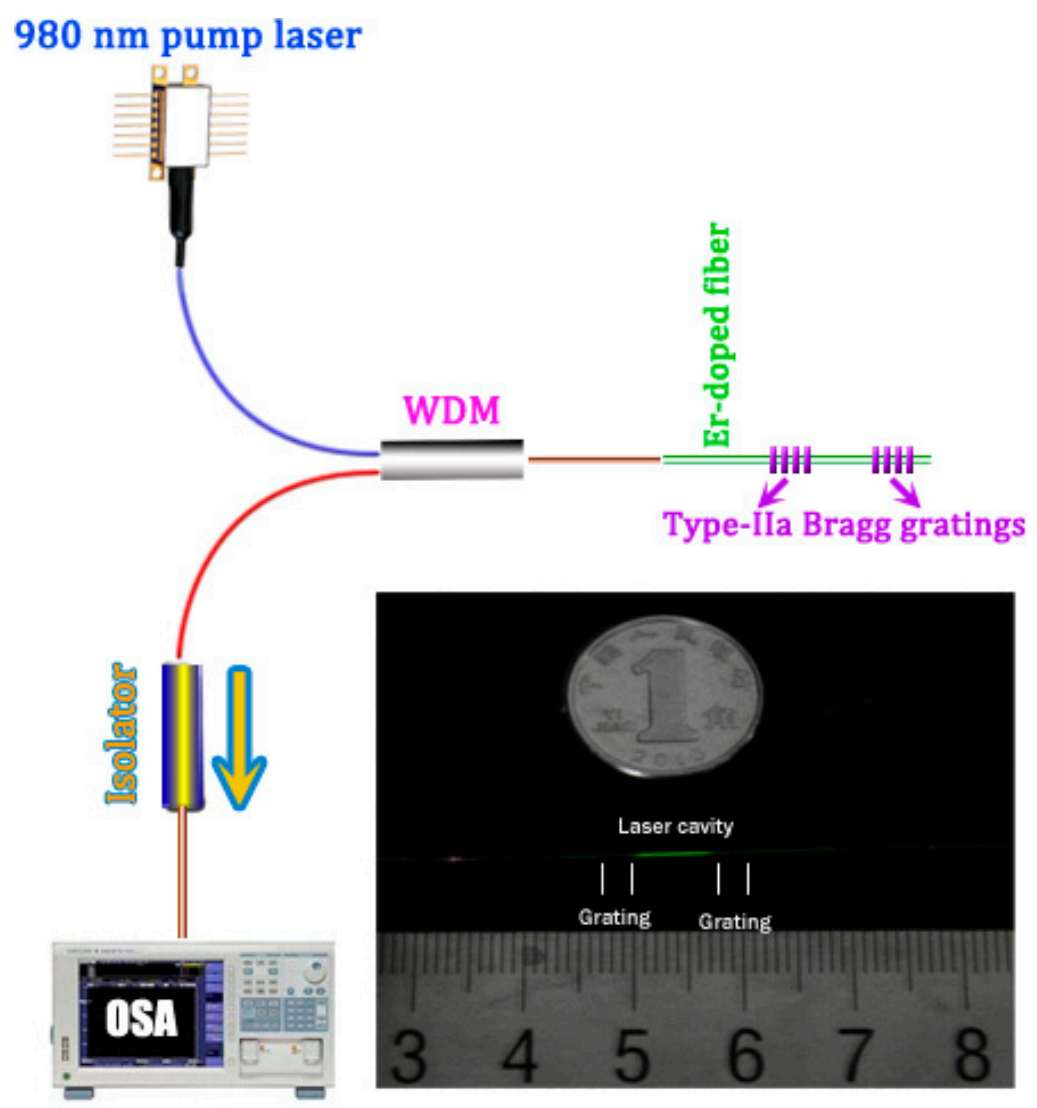

Figure 5. Schematic of the DBR laser structure. Inset: the photograph of the DBR laser. WDM: wavelength division multiplexer.

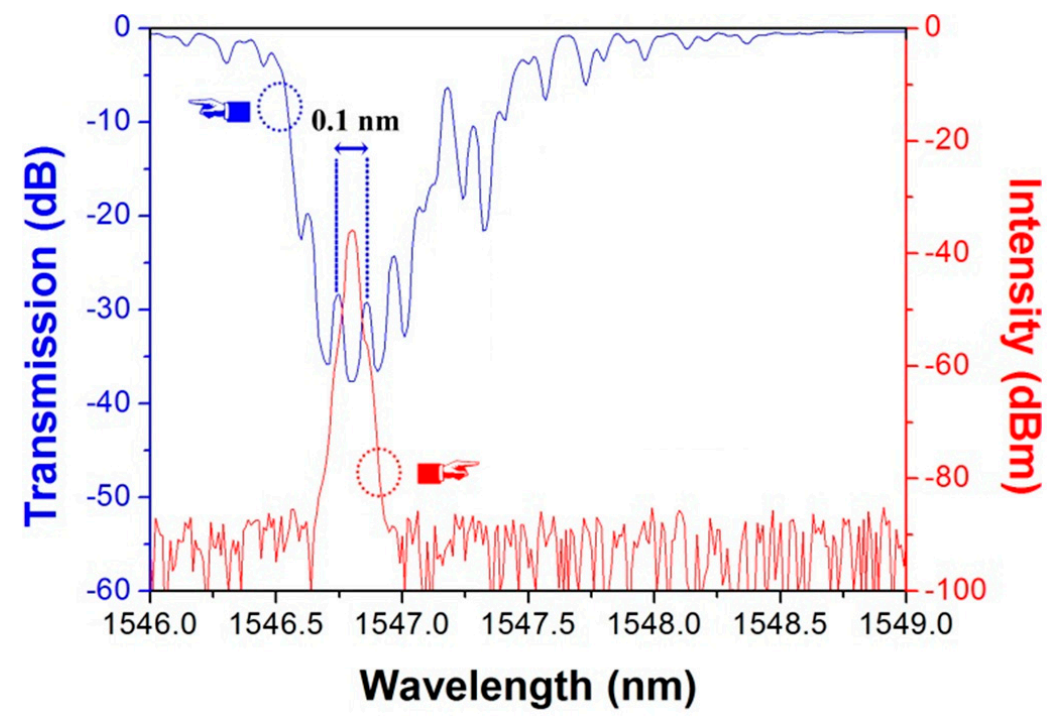

Figure 6. Spectra of the F-P interference and DBR laser output. 

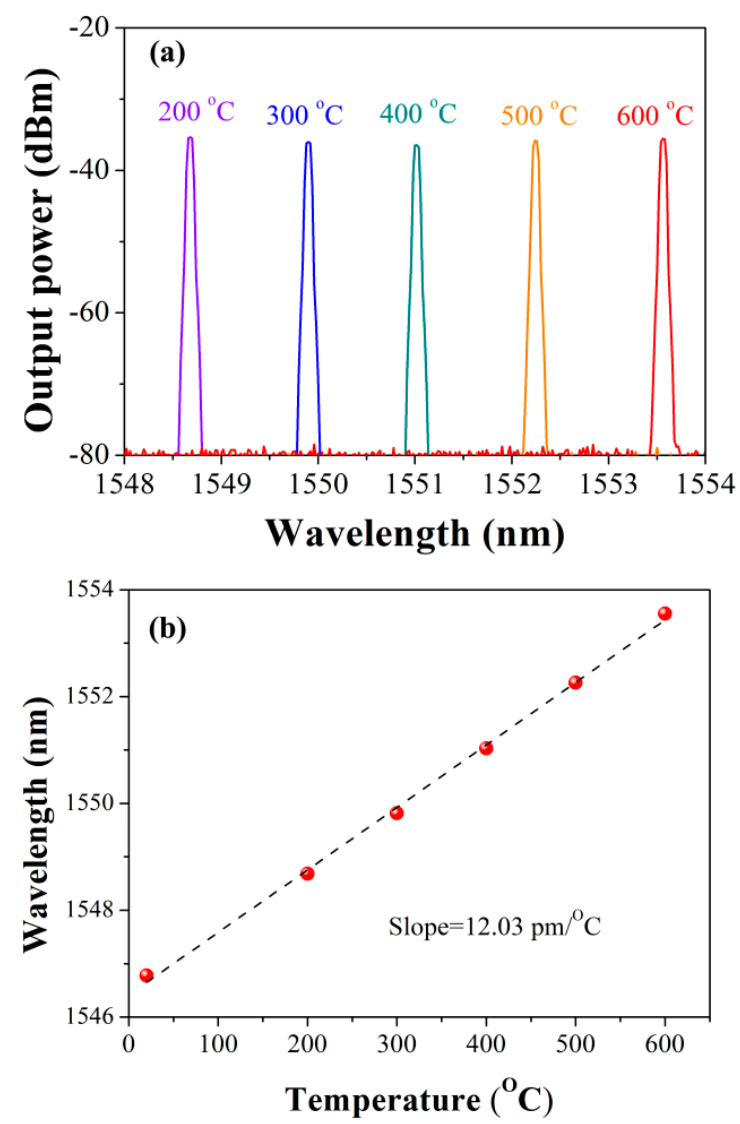

Figure 7. (a) Measured output spectra of the laser at different temperatures; and (b) lasing wavelength versus temperature.

The stability of laser output power at high temperature of $600{ }^{\circ} \mathrm{C}$ is presented in Figure 8. The average power is $-35.5 \mathrm{dBm}$ with standard deviation of $\sim 0.18$. Therefore, it can be concluded that the Type-IIa grating based DBR fiber laser shows good resistance to $600{ }^{\circ} \mathrm{C}$, enabling its potential of acting laser sensors applied in harsh environment and laser source with high stability, as well as a large dynamic tuning range of wavelengths [23].

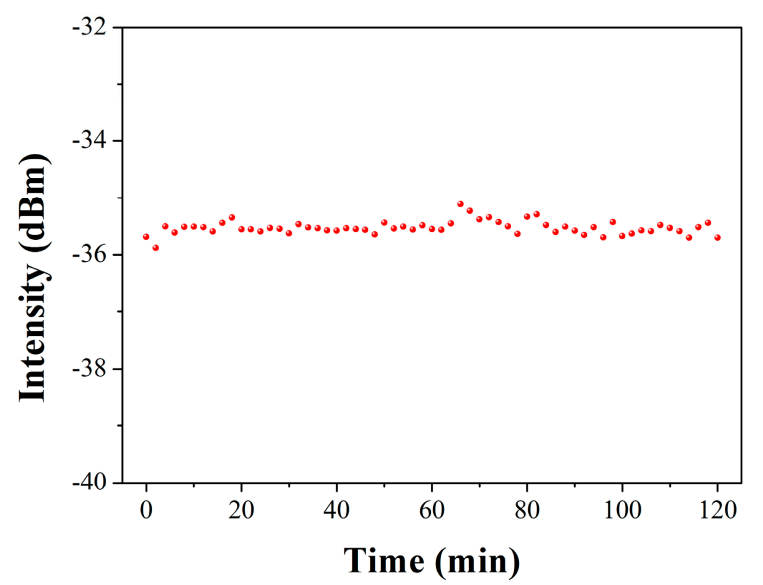

Figure 8. Stability test result of the laser at $600^{\circ} \mathrm{C}$ for $2 \mathrm{~h}$. 


\subsection{Thermally-Triggered DBR Fiber Laser}

If the UV exposure ends just before the grating integration, the two dips arising from the secondary grating and Type-IIa resonance remain separated.

Figure 9 shows the temperature response of a two-dip grating with an initial wavelength separation of $0.28 \mathrm{~nm}$. The transmission spectrum of the grating is recorded at different temperatures from room temperature to $600{ }^{\circ} \mathrm{C}$. The secondary resonance which locates at short wavelength side shows a higher temperature sensitivity than the Type-IIa resonance. Therefore, the wavelength separation decreases with temperature, and the two dips tend to merge with each other as temperature increases. When the temperature is increased to $500^{\circ} \mathrm{C}$, the two dips integrate entirely. As a result, the grating reflectivity is strongly enhanced due to the overlapping of the two grating components.

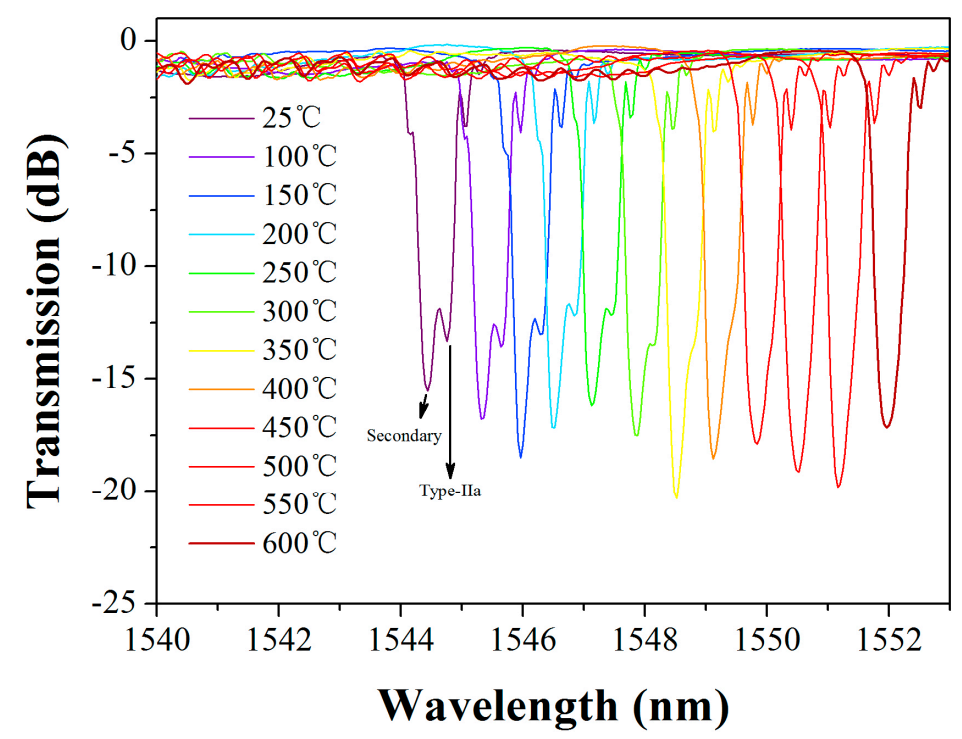

Figure 9. Measured transmission spectra of a two-dip grating at different temperatures.

The characteristic of those dual-dip gratings provide the opportunity to form a DBR fiber laser whose threshold can be thermally tuned. A pair of such two-dip gratings is inscribed at both end of a piece of Er-doped fiber with certain spacing. The cavity length is $17 \mathrm{~mm}$. A $980 \mathrm{~nm}$ pump laser with output power tunable from 0 to $500 \mathrm{~mW}$ is connected to the laser cavity. Thus, the thresholds of the DBR laser can be measured by tuning the pump power at different temperatures as shown in Figure 10.

It can be observed that the pump threshold varies with the temperature. As the temperature increases to $250{ }^{\circ} \mathrm{C}$, the threshold is significantly reduced to less than $70 \mathrm{~mW}$ indicating that the secondary dip starts to overlap with the Type-IIa dip. Thus, higher reflectivity of the gratings are enabled. Higher temperature yields lower pump power for laser oscillation because the two dips are better overlapped. At $450{ }^{\circ} \mathrm{C}$, the threshold reaches the minimum value, just a $30 \mathrm{~mW}$ pump power is enough to establish the laser output. Here, given that the intensity of the ultra-short DBR laser maintains during the temperature increasing which is shown in Figure 7a of Section 3.1, the variation of the laser threshold in this case is mainly attributed to the grating reflectivity enhancement.

Figure 10 shows two regions denoted as "Output area" and "Non-output area", divided by the threshold curve. If the combination of pump power and temperature falls in the output area, the laser can be triggered. Otherwise, there is no laser output. However, if we set the combination in the non-output area, but close to the thresholds of the temperature above $150{ }^{\circ} \mathrm{C}$, the laser can be triggered by increasing the temperature while the pump power is maintained. For example, as shown in Figure 10, as the pump power is set to $60 \mathrm{~mW}$, the laser cannot be triggered if the temperature is below $250{ }^{\circ} \mathrm{C}$. However, if we increase the temperature to $300^{\circ} \mathrm{C}$, the laser can be triggered. With the further increase of temperature, the laser power is accordingly enhanced due to the strengthening of 
the grating reflectivity. As the temperature increases to $450{ }^{\circ} \mathrm{C}$, the highest power can be obtained, up to $-40 \mathrm{dBm}$, probably caused by the annealing of the secondary dip as the temperature is higher than $450{ }^{\circ} \mathrm{C}$. Nevertheless, depending on the high temperature resistance of Type-IIa grating, those thermally-triggered DBR fiber lasers can be potentially used as high-temperature alarms, of whose warning temperature can be flexibly managed through setting the pump power [27].
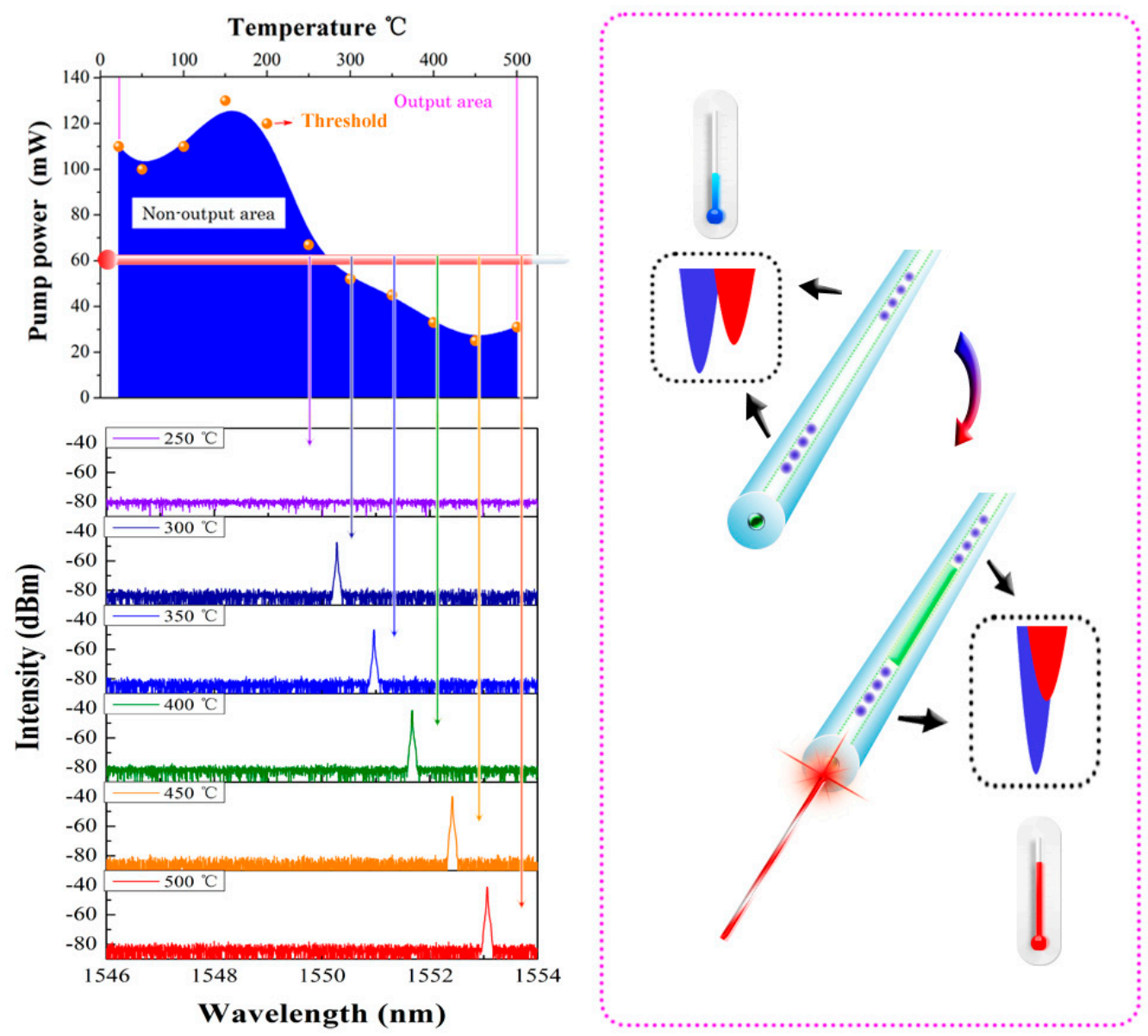

Figure 10. Pump threshold and the thermal trigger (left) of the two-dip grating based DBR fiber laser (right).

\subsection{Third Harmonic Grating Fiber Laser Operating at 1- $\mu m$}

The larger index change contrast of the two interleaving modulation structures which is essential in forming secondary grating can also lead to a high strength resonance at the third harmonic wavelength.

The resonant wavelengths of the FBG can be expressed as [25,28] in Equation (1):

$$
\lambda(j)=\frac{2}{j} n_{e f f} \Lambda
$$

where $\lambda(j)$ is the reflection wavelength at the harmonic orders $j=1,2,3, \ldots, \Lambda$ denotes the grating period and $n_{\text {eff }}$ is the effective index. For an ideal phase mask, only the \pm 1 diffraction orders of the UV light exist, the Bragg wavelength $\lambda_{B}$ is the first harmonic reflection $(j=1)$ and $\Lambda$ is half of the pitch of the phase mask $\left(\Lambda_{\mathrm{pm}}\right)$. For practical cases, the participation of the \pm 2 diffraction orders results in Talbot-type fringes, two modulating structures which are both dominated by $\Lambda=\Lambda_{\text {pm }}$ interleave into the fiber core. $\lambda_{B}$ is primarily attributed to the second harmonic resonance $(j=2)$. Therefore, it can be predicted that a strong dip would appear at the wavelength around $1 \mu \mathrm{m}$ in accordance 
with the third harmonic resonance. We monitor the evolution of this secondary-Type-IIa grating in $1 \mu \mathrm{m}$ and $1.5 \mu \mathrm{m}$ wavelength-windows simultaneously, as shown in Figure 11. At the wavelength of $\sim 1039 \mathrm{~nm}$, a resonance dip can be observed in all of the growing stages of the secondary-Type-IIa grating. Note that there is only one dip other than the double dips at $2 / 3 \lambda_{B}$ which was reported in [17] because only a half Talbot-layer is supported in the fiber core, avoiding the $\pi$-phase shift structures [29]. At the end of the primary grating decay stage, the $2 / 3 \lambda_{B}$ resonance reaches the maximum strength with reflectivity higher than $95 \%$, denoting the largest overall photorefractive effect contrast between the two modulating structures. Subsequently, the third-harmonic-dip decays gradually along with the integrating of the secondary and Type-IIa dips, unveiling the decrease of the overall photorefractive effect contrast. This result highly affirms that the Talbot-type-fringes are the dominating factor in the formation of the SBG.

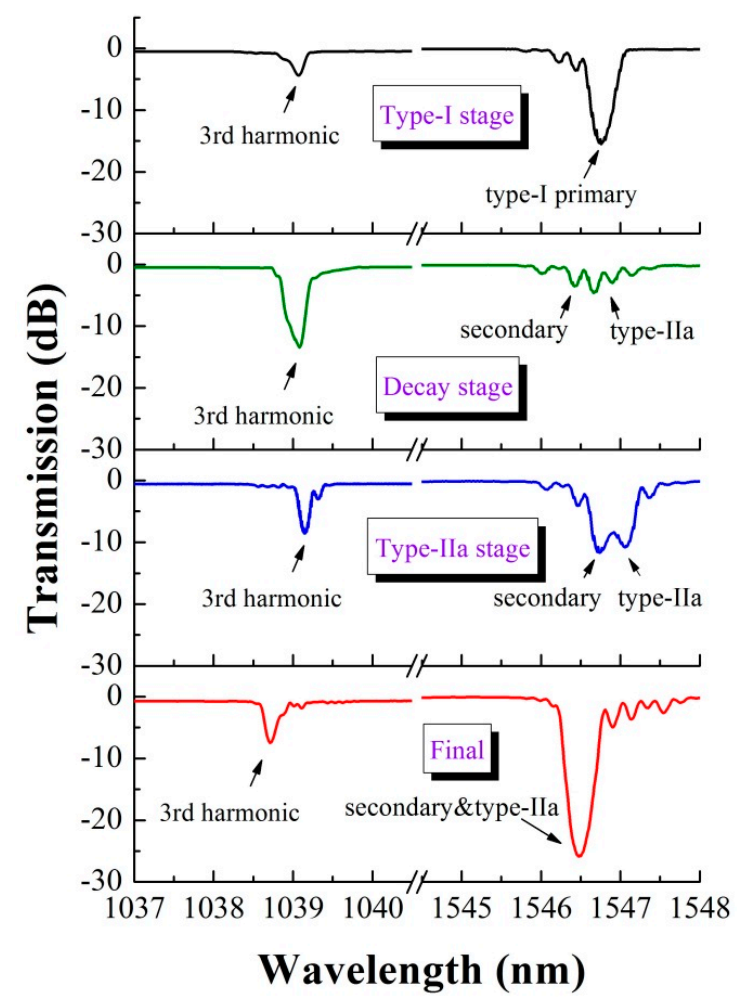

Figure 11. Spectral evolution of the "secondary-Type-IIa" grating at $1 \mu \mathrm{m}$ and $1.5 \mu \mathrm{m}$ bands.

As a sequence, those gratings can be applied as Bragg reflectors operating at $1-\mu \mathrm{m}$-wavelength, taking advantage of the high reflection of the third harmonic resonance [30]. We fabricate two wavelength-matched gratings in a photosensitive fiber and then splice them to a $20 \mathrm{~mm}$ length of ytterbium $(\mathrm{Yb})$-doped single-mode active fiber to form a Fabry-Perot cavity with an interference spectrum in a 1- $\mu \mathrm{m}$-wavelength window. The $\mathrm{Yb}$-doped fiber has core and cladding diameters of $\sim 3.5 \mu \mathrm{m}$ and $125 \mu \mathrm{m}$, respectively. The numerical aperture (NA) is 0.18 . The numerical aperture (NA) is 0.18 . By connecting a $980 \mathrm{~nm}$ pump laser, the DBR laser operated at a wavelength of $1039 \mathrm{~nm}$ can be formed by virtue of the strong reflectors and the high pump absorption of $1200 \mathrm{~dB} / \mathrm{m}$ in the $\mathrm{Yb}$-doped fiber. By tuning the $980 \mathrm{~nm}$ pump laser from 0 to $100 \mathrm{~mW}$, the output characteristics of the third harmonic DBR laser can be measured, which is shown in Figure 12. The laser presents a positive response to the pump power, whose pump threshold is $20 \mathrm{~mW}$. At pump power of $100 \mathrm{~mW}$, the laser can output power over $1 \mathrm{~mW}$. During the pump-tuning period, the laser wavelength stably stays at $1039.1 \mathrm{~nm}$, due to the lower pump absorption of the grating region $(5 \mathrm{~dB} / \mathrm{m})$. 


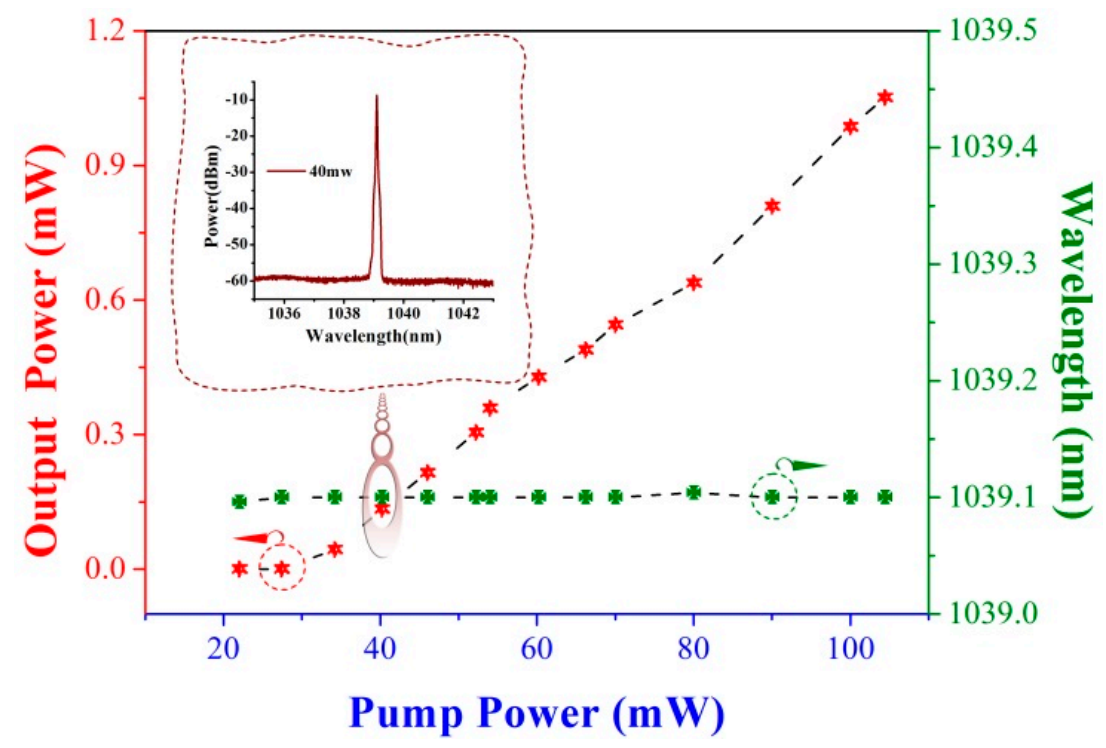

Figure 12. Changes of the power and wavelength of the laser versus pump power. Inset: the laser spectrum at pump power of $40 \mathrm{~mW}$.

Maintaining the pump power at $40 \mathrm{~mW}$, the laser is used in the following study, whose spectrum is displayed in the inset of Figure 12. At first, the strain response of the third harmonic DBR laser is measured by loading an axial tension onto both sides. By increasing the tension gradually, the correlation between the strain and laser wavelength can be obtained. According to Figure 13a, the strain sensitivity is $0.63 \mathrm{pm} / \mu \varepsilon$. Then the temperature response of the laser is tested. From room temperature to $100{ }^{\circ} \mathrm{C}$, the correlation between the temperature and laser wavelength is shown in Figure $13 \mathrm{~b}$. The temperature sensitivity is $6.3 \mathrm{pm} /{ }^{\circ} \mathrm{C}$. The results are highly coincident with the sensing characteristics of the third harmonic gratings [17]. The sensitivities corresponding to the temperature and strain are significantly lower than those of $1550 \mathrm{~nm}$ DBR laser [31]. Therefore, the third harmonic DBR fiber laser can present higher stability in wavelength depending on the lower disturbance influenced by the strain and temperature.

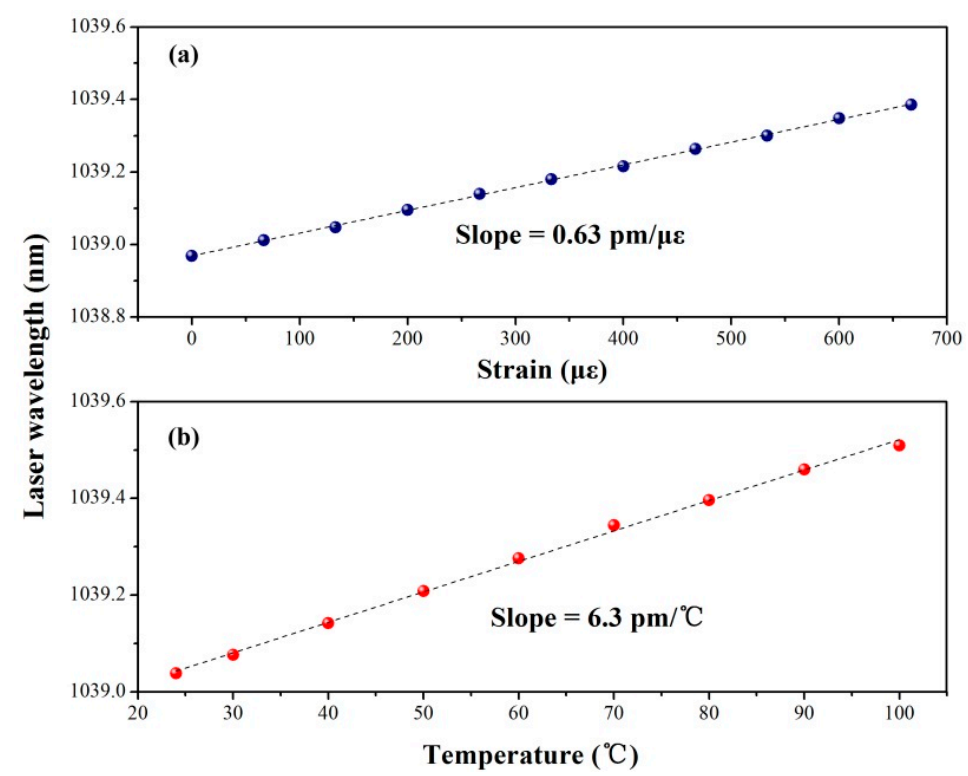

Figure 13. Laser sensitivity of the wavelength responding to the (a) strain or (b) temperature. 
The long term wavelength-stability of the laser is tested at different temperatures. From Figure 14, the wavelength variation is less than $2 \times 10^{-6} \mathrm{~nm}$ at each temperature over two hours. As a sequence, a wavelength-reliable DBR laser operating at $1 \mu \mathrm{m}$ can be presented providing the potential in optical lattice clocks, space probes, and sensors [32-35].

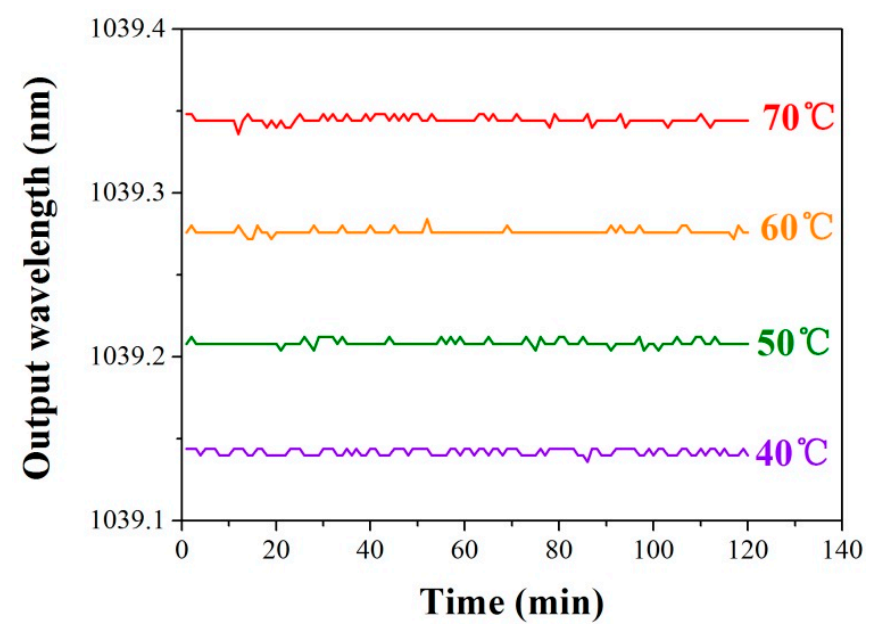

Figure 14. Long-term stability of the lasing wavelength at different temperatures.

Furthermore, an extensional thought should emphasize on the Talbot-type-fringes. Compared with other frequently used inscription laser with wavelengths of $244 \mathrm{~nm}$ or $248 \mathrm{~nm}$, the $193 \mathrm{~nm}$ laser not only provides higher index modulation efficiency without hydrogen-loading but also supports a larger Talbot length. Thus, a single-layer modulation structure in the fiber core can be realized with greater possibility, which is important to form higher order harmonic reflection in the grating. It can be estimated that phase mask with pitch as low as $1044 \mathrm{~nm}$, which results in a Bragg wavelength of $1513 \mathrm{~nm}$, can still guarantee the Talbot length larger than $6 \mu \mathrm{m}$, twice the fiber core that we used. Therefore, the phase masks designed for $1.5 \mu \mathrm{m}$ waveband gratings can be utilized in fabricating third harmonic lasers operating at the wavelength within the gain spectrum of the Yb-doped fibers. Thus, the secondary grating formation mechanism offers great flexibility for designing the wavelength of the fiber lasers.

\section{Conclusions}

In this paper, a unique grating named the secondary Bragg grating is presented. Depending on the Talbot-type-fringes of the inscription light, two interleaving modulation structures would be formed along the fiber axis. A large contrast of the index change exists as if the diameter of the photosensitive core is equal to half of the Talbot length. By a large amount of the cumulative fluence, which is needed in Type-IIa FBG inscription, the weaker periodical modulation would result in the generating of the SBG. Sensing applications of the DBR fiber lasers based on those SBGs are demonstrated. For instance, a DBR fiber laser with ultra-short dimension can act as a compact temperature sensor, even in extreme temperature environments; a threshold-tunable DBR fiber laser can be utilized as the flexibly-managed high-temperature alarm; the third harmonic reflector-based fiber laser presents an anti-jamming 1- $\mu \mathrm{m}$ wavelength output, providing the potential in space probing and coherent optical detection. Given that the investigation of this new type of grating is still underway, the future results may further enrich the study and the application development of the FBG devices.

Acknowledgments: This work is supported by National Natural Science Foundation of China (Grant No. 61405074), Guangdong Natural Science Foundation (Grant Nos. 2015A030313324, S2013030013302), Pearl River S\&T Nova Program of Guangzhou (Grant No. 201610010151) and “Climbing Program” Special Funds (Grant No. pdjh2016a0051). 
Author Contributions: Bai-Ou Guan and Yang Ran conceived and designed the experiments; Fu-Rong Feng performed the experiments; Yang Ran and Long Jin analyzed the data; Long Jin contributed reagents/ materials/analysis tools; Bai-Ou Guan and Yang Ran wrote the paper.

Conflicts of Interest: The authors declare no conflict of interest.

\section{References}

1. Hill, K.O.; Meltz, G. Fiber Bragg grating technology fundamentals and overview. J. Lightwave Technol. 1997, 15, 1263-1276. [CrossRef]

2. Morey, W.W.; Meltz, G.; Glenn, W.H. Fibre optic Bragg grating sensors. Proc. SPIE 1989, 1169, $98-107$.

3. Rao, Y.J.; Ribeiro, A.B.L.; Jackson, D.A.; Zhang, L.; Bennion, I. Combined spatial- and time-division-multiplexing scheme for fibre grating sensors with drift-compensated phase-sensitive detection. Opt. Lett. 1995, 20, 2149-2151. [CrossRef] [PubMed]

4. Shao, L.Y.; Xiong, L.Y.; Chen, C.K.; Laronche, A.; Albert, J. Directional bend sensor based on re-grown tilted fiber Bragg grating. J. Lightwave Technol. 2010, 28, 2681-2687. [CrossRef]

5. Guo, T.; Shang, L.B.; Ran, Y.; Guan, B.O.; Albert, J. Fiber-optic vector vibroscope. Opt. Lett. 2012, 37, 2703-2705. [CrossRef] [PubMed]

6. Fisher, N.E.; Webb, D.J.; Pannell, C.N.; Jackson, D.A.; Gavrilov, L.R.; Hand, J.W.; Zhang, L.; Bennion, I. Ultrasonic hydrophone based on short in-fiber Bragg gratings. Appl. Opt. 1998, 37, 8120-8128. [CrossRef] [PubMed]

7. Ran, Y.; Tan, Y.N.; Sun, L.P.; Gao, S.; Li, J.; Jin, L.; Guan, B.O. 193 nm excimer laser inscribed Bragg gratings in microfibers for refractive index sensing. Opt. Express 2011, 19, 18577-18583. [CrossRef] [PubMed]

8. Sun, D.D.; Guo, T.; Ran, Y.; Huang, Y.Y.; Guan, B.O. In-situ DNA hybridization detection with a reflective microfiber grating biosensor. Biosens. Bioelectron. 2014, 61, 541-546. [CrossRef] [PubMed]

9. Canning, J. Fibre gratings and devices for sensors and lasers. Laser Photon. Rev. 2008, 2, 275-289. [CrossRef]

10. Mihailov, S.J. Fiber Bragg grating sensors for harsh environments. Sensors 2012, 12, 1898-1918. [CrossRef] [PubMed]

11. Cordier, P.; Dupont, S.; Douay, M.; Martinelli, G.; Bernage, P.; Niay, P.; Bayon, J.; Dong, L. Evidence by transmission electron microscopy of densification associated to Bragg grating photoimprinting in germanosilicate optical fibers. Appl. Phys. Lett. 1997, 70, 1204-1206. [CrossRef]

12. Dianov, E.; Plotnichenko, V.; Koltashev, V.; Pyrkov, Y.N.; Ky, N.; Limberger, H.; Salathe, R. UV-irradiation-induced structural transformation of germanoscilicate glass fiber. Opt. Lett. 1997, 22, 1754-1756. [CrossRef] [PubMed]

13. Dong, L.; Liu, W.F.; Reekie, L. Negative-index gratings formed by a 193-nm excimer laser. Opt. Lett. 1996, 21, 2032-2034. [CrossRef] [PubMed]

14. Riant, I.; Haller, F. Study of the photosensitivity at $193 \mathrm{~nm}$ and comparison with photosensitivity at $240 \mathrm{~nm}$ influence of fiber tension: Type IIa aging. J. Lightwave Technol. 1997, 15, 1464-1469. [CrossRef]

15. Ky, N.H.; Limberger, H.G.; Salath, R.P.; Cochet, F.; Dong, L. UV-irradiation induced stress and index changes during the growth of Type-I and Type-IIa fiber gratings. Opt. Commun. 2003, 225, 313-318. [CrossRef]

16. Groothoff, N.; Canning, J. Enhanced type IIa gratings for high-temperature operation. Opt. Lett. 2004, 29, 2360-2362. [CrossRef] [PubMed]

17. Rollinson, C.; Wade, S.A.; Dragomir, N.; Baxter, G.W.; Collins, S.F.; Roberts, A. Reflections near $1030 \mathrm{~nm}$ from $1540 \mathrm{~nm}$ fibre Bragg gratings: Evidence of a complex refractive index structure. Opt. Commun. 2005, 256, 310-318. [CrossRef]

18. Shu, X.; Liu, Y.; Zhao, D.; Gwandu, B.; Floreani, F.; Zhang, L.; Bennion, I. Dependence of temperature and strain coefficients on fiber grating type and its application to simultaneous temperature and strain measurement. Opt. Lett. 2002, 27, 701-703. [CrossRef] [PubMed]

19. Violakis, G.; Konstantaki, M.; Pissadakis, S. Accelerated recording of negative index gratings in Ge-doped optical fibers using 248-nm 500-fs laser radiation. IEEE Photon. Technol. Lett. 2006, 18, 1182-1184. [CrossRef]

20. Pissadakis, S.; Livitziis, M.; Tsibidis, G.D.; Kobelke, J.; Schuster, K. Type Ila grating inscription in a highly nonlinear microstructured optical fiber. IEEE Photon. Technol. Lett. 2009, 21, 227-229. [CrossRef]

21. Prakash, O.; Kumar, J.; Mahakud, R.; Agrawal, S.K.; Dixit, S.K.; Nakhe, S.V. Enhanced temperature $\left(\sim 800^{\circ} \mathrm{C}\right)$ stability of Type-IIa FBG written by $255 \mathrm{~nm}$ beam. IEEE Photon. Technol. Lett. 2014, 26, 93-95. [CrossRef] 
22. Ran, Y.; Jin, L.; Gao, S.; Sun, L.P.; Huang, Y.Y.; Li, J.; Guan, B.O. Type Ila Bragg gratings formed in microfibers. Opt. Lett. 2015, 40, 3802-3805. [CrossRef] [PubMed]

23. Ran, Y.; Feng, F.R.; Liang, Y.Z.; Jin, L.; Guan, B.O. Type IIa Bragg grating based ultra-short DBR fiber laser with high temperature resistance. Opt. Lett. 2015, 40, 5706-5709. [CrossRef] [PubMed]

24. Dyer, P.; Farley, R.; Giedl, R. Analysis of grating formation with excimer laser irradiated phase masks. Opt. Commun. 1995, 115, 327-334. [CrossRef]

25. Rollinson, C.M.; Wade, S.A.; Kouskousis, B.P.; Kitcher, D.J.; Baxter, G.W.; Collins, S.F. Variations of the growth of harmonic reflections in fiber Bragg gratings fabricated using phase masks. J. Opt. Soc. Am. A 2012, 29, 1259-1268. [CrossRef] [PubMed]

26. Mills, J.D.; Hillman, C.W.J.; Blott, B.H.; Brocklesby, W.S. Imaging of free-space interference patterns used to manufacture fiber Bragg gratings. Appl. Opt. 2000, 39, 6128-6135. [CrossRef] [PubMed]

27. Feng, F.R.; Ran, Y.; Liang, Y.Z.; Gao, S.; Feng, Y.H.; Jin, L.; Guan, B.O. Thermally triggered fiber lasers based on secondary-type In Bragg gratings. Opt. Lett. 2016, 41, 2470-2473. [CrossRef] [PubMed]

28. Erdogan, T. Fiber grating spectra. J. Lightwave Technol. 1997, 15, 1277-1294. [CrossRef]

29. Yam, S.P.; Brodzeli, Z.; Kouskousis, B.P.; Rollinson, C.M.; Wade, S.A.; Baxter, G.W.; Collins, S.F. Fabrication of a $\pi$-phase-shifted fiber Bragg grating at twice the Bragg wavelength with the standard phase mask technique. Opt. Lett. 2009, 34, 2021-2023. [CrossRef] [PubMed]

30. Feng, F.R.; Liu, T.; Xiao, P.; Ran, Y.; Liang, H.; Jin, L.; Guan, B.O. 1- $\mu$ m-wavelength ytterbium-doped fiber laser based on the third harmonic reflection in secondary-Type-In Bragg gratings. Opt. Lett. 2016, 41, 4999-5002. [CrossRef] [PubMed]

31. Wo, J.; Jiang, M.; Malnou, M.; Sun, Q.; Zhang, J.; Shum, P.P.; Liu, D. Twist sensor based on axial strain insensitive distributed Bragg reflector fiber laser. Opt. Express 2012, 20, 2844-2850. [CrossRef] [PubMed]

32. Babin, S.A.; Churkin, D.V.; Ismagulov, A.E.; Kablukov, S.I.; Nikulin, M.A. Single frequency single polarization DFB fiber laser. Laser Phys. Lett. 2007, 4, 428-432. [CrossRef]

33. Xu, S.; Yang, Z.; Zhang, W.; Wei, X.; Qian, Q.; Chen, D.; Zhang, Q.; Shen, S.; Peng, M.; Qiu, J. 400 mW ultrashort cavity low-noise single-frequency $\mathrm{Yb}^{3+}$-doped phosphate fiber laser. Opt. Lett. 2011, 36, 3708-3710. [CrossRef] [PubMed]

34. Mo, S.; Xu, S.; Huang, X.; Zhang, W.; Feng, Z.; Chen, D.; Yang, T.; Yang, Z. A 1014 nm linearly polarized low noise narrow-linewidth single-frequency fiber laser. Opt. Express 2013, 21, 12419-12423. [CrossRef] [PubMed]

35. Xu, S.; Li, C.; Zhang, W.; Mo, S.; Yang, C.; Wei, X.; Feng, Z.; Qian, Q.; Shen, S.; Peng, M.; et al. Low noise single-frequency single-polarization ytterbium-doped phosphate fiber laser at $1083 \mathrm{~nm}$. Opt. Lett. 2013, 38, 501-503. [CrossRef] [PubMed]

(C) 2017 by the authors; licensee MDPI, Basel, Switzerland. This article is an open access article distributed under the terms and conditions of the Creative Commons Attribution (CC BY) license (http:/ / creativecommons.org/licenses/by/4.0/). 